\title{
Search for diffuse $\gamma$-ray emission from galactic plane with YangBaJing Hybrid Array
}

\section{Yuhua Yao ${ }^{* a b}$, Jiancheng $\mathrm{He}^{b}$, Yiqing Guo ${ }^{b}$, Yi Zhang ${ }^{b}$, Cheng Liu ${ }^{b}$, Tianlu Chen ${ }^{c}$ and Hongbo $\mathrm{Hu}^{b}$}

${ }^{a}$ College of Physical Science and Technology, Sichuan University

N.29 Wangjiang Road, Jiuyanqiao, Wuhou District, Chengdu City, Sichuan Province, 610064,

P.R. China

${ }^{b}$ Institute of High Energy Physics, Chinese Academy of Sciences

No.19B Yuquan Road, Shijingshan District, Beijing, 100049, P.R. China

${ }^{c}$ Physics Department of Science School, Tibet University

N.10, Zandadonglu, Chengguan District, Lhasa, Tibet autonomous region, 850000, P.R. China

E-mail: vaovhdihep.ac.cn, hejcdihep.ac.cn, guovg.ihep.ac.cn,

zhangyidihep.ac.cn liucdihep.ac.cn,

chentldihep.ac.cn,huhbeihep.ac.cn

\begin{abstract}
As one of the pilot experiments of LHAASO, a hybrid array (HA) covering an area of $20000 \mathrm{~m}^{2}$ was successfully constructed by the end of 2016 at the international Cosmic Ray Observatory at YangBajing (YBJ) in Tibet of China. This array consists of 115 scintillation detectors and 16 underground muon detectors of $900 \mathrm{~m}^{2}$. Using muons information, most of hadronic air showers are rejected at several dozens of $\mathrm{TeV}$ energy regime. With the data collected from 2017 to 2018, this work presents preliminary results on diffuse $\gamma$-ray emission from galactic plane. As a result, there has no significant excess of $\mathrm{TeV} \gamma$-ray in the galactic plane with the YBJ-HA observation. Therefore, we put a 90\% CL upper limit. We foresee that the under-construction LHAASO will have great potential in observing $\gamma$-rays in several dozens of even up to hundreds of $\mathrm{TeV}$ energy range.
\end{abstract}

36th International Cosmic Ray Conference -ICRC2019-

July 24th - August 1st, 2019

Madison, WI, U.S.A.

\footnotetext{
* Speaker.
} 


\section{Introdution}

Diffuse $\gamma$-ray is considered to be produced in the interactions of Cosmic Rays (CRs) with the interstellar matter (ISM) and radiation fields (ISRF). Since $\gamma$-rays wouldn't be affected by the magnetic field during their propagation, diffuse $\gamma$-ray is of importance to study the density of the ISM, the propagation of CRs, and the characteristics of ISRF . Last decades have witnessed the development of the observation of diffuse $\gamma$-ray, examples include OSO-III[W], SAS-2[], EGRET[B],

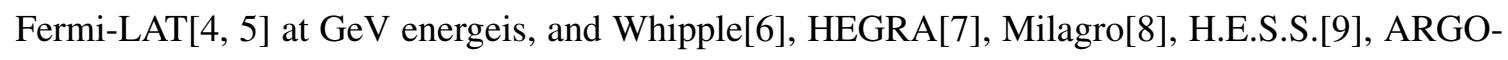
YBJ[ए]] at TeV energies.

The gas density of galactic plane is very intensive, therefore this region is a compromising target for the study of diffuse $\gamma$-ray emissions. In this area, Milagro telescope performed the observations of CYGNUS region and found the diffuse $\mathrm{TeV} \gamma$-ray emission[[]]. Subsequently ARGOYBJ experiment carried out a similar observation [ए]], which agreed well with the measurements of Fermi-LAT at lower energy. Meanwhile, H.E.S.S. telescope array also performed survey at both galactic plane[Q] and center[Ш], [2]. Additionally, IceCube has searched for diffuse $\gamma$-ray in PeV energy range but didn't find significant excess [[13] .

To date, diffuse $\gamma$-ray in the galactic plane has been measured up to TeV energies. The YBJHA, benefitting by its high altitude which is about 4300 meter above sea level and large FOV of about 2 steradian, is probable to capture some higher energies $\gamma$-ray emissions in this region. This work presents the preliminary results of the analysis of the diffuse $\gamma$-ray emission from galactic plane with energy above several dozens of $\mathrm{TeV}$.

\section{Experiment}

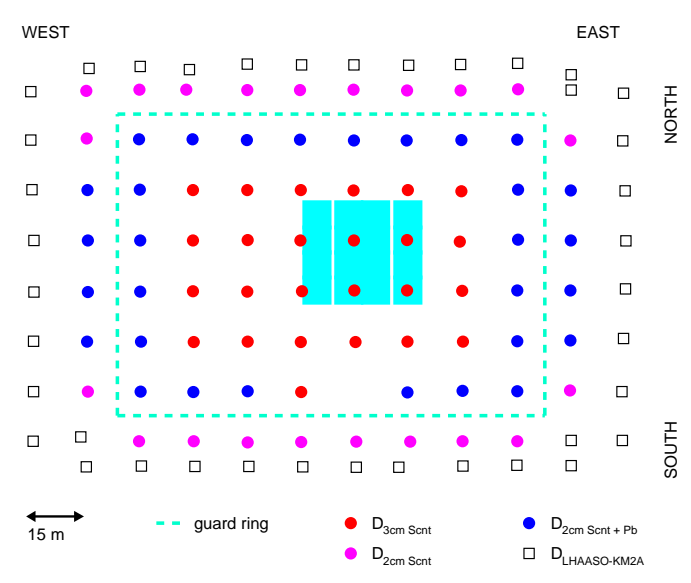

Figure 1: The schematic view of the YBJ-HA.

YBJ-HA is located at the site of the International YBJ observatory, which hosts the well known $\mathrm{AS} \gamma$ and ARGO-YBJ experiments. This is an altitude where the extensive air showers reach the maximum when their energy approach PeV. Fig. 1 is a schematic view of the array. It consists of 115 scintillation surface detectors (SDs) which are used to detect the electromagnetic components, 
and 16 underground water Cherenkov muon detectors (MDs) about $900 \mathrm{~m}^{2}$ focusing on observing the muon component. Since CRs have hundred times more muon number than that of $\gamma$-rays, the detection of muon is an effective tool to distinct $\gamma$-ray events from the enormous CRs background. SDs are sparse detectors spaced 15 meters apart, while MDs consists of 16 compact water cells. More detailed information about the array is presented in the Ref.[प4]].

In order to get a better understanding of our data, along with the analysis of data, we perform the Monte carlo (MC) simulation with Corsika-74005 and Geant4.09.03. In the simulation of CRs, the hadronic models QGSJET2 and GHEISHA are adopted for high and low energy respectively. Five groups of nuclei are simulated, including $\mathrm{H}, \mathrm{He}, \mathrm{CNO}, \mathrm{MgAlSi}, \mathrm{Fe}$, then normalized into observed spectrums according to Gasser[ए5] and Horandel[ए]6] component models. The sample of $\gamma$-ray is generated by a spectrum index of -2.59 , and it can be scaled to any specific spectrum. Geant 4 are used to simulate the process of charge particles and photons in SDs and MDs, to record variables such as arrival time and deposition energy. The same reconstruction procedures are used for MC samples and experimental data.

\section{Analysis}

\section{1 data selection}

The data set used in this work is taken from January 2017 to June 2018, totally $\sim 160$ days of live time because most of the time was devoted to debugging the White Rabbit electronic system of LHAASO. With the MC study, the threshold energy of the dataset is about several TeV. According to the characteristic of the reconstructed events, to ensure the quantity of the analyzed dataset, we choose those events that meet the following selection criteria:

1. reconstructed zenith angle is $\theta \leq 45^{\circ}$;

2. the estimated core location is inside the guard ring;

3. the residual of time fitting is $\leq 1.0 \mathrm{~m}$;

4. sum of the particle's density (sumpd), which is the primary energy estimator, is $\geq 100$;

5. muon-poor event, whose total muon number $N_{\mu}<0.6$, is considered as $\gamma$-like event.

After event selections, the total analysed data samples is about $3 \times 10^{5}$, and according to the MC study, the median primary energy of $\gamma$-ray is $\sim 50 \mathrm{TeV}$ and the angular resolution is $\sim 0.7^{\circ}$.

\section{2 background estimation}

The diffuse $\gamma$-rays from the galactic plane, including inner $\left(|g b| \leq 3^{\circ}, 20^{\circ} \leq g l \leq 105^{\circ}\right)$ and outer $\left(|g b| \leq 3^{\circ}, 140^{\circ} \leq g l \leq 225^{\circ}\right)$ plane, are targets of this work. The muon-poor selection has been applied to distinguish $\gamma$-ray signal from overwhelming CR background. However, it's no doubt that there are still CR background contamination in the muon-poor samples. Therefore, we adopt the All-Distance Equi-Zenith Method introduced in [ㄱ] to estimate the number of background in the signal region. Unlike traditional Equi-Zenith Method, which is often used to estimate 
the background for the point gamma-ray source, this method is fit for large-scale signal or background estimation, which meets the characteristics of this work.

The celestial space is divided into small cells $(i, j)$ with $2^{\circ} \times 2^{\circ}$, from $0^{\circ}$ to $360^{\circ}$ in right ascension and from $0^{\circ}$ to $60^{\circ}$ in declination. One thing to note is that, the bin size cell is much larger than the the angular resolution of the array in order to avoid empty grid. While in the observer's horizontal coordinates, the zenith is divided by a step of $2^{\circ}$, and the azimuth angle is binned dependent on the zenith, the step is $2^{\circ} / \sin (\theta)$. The relative intensity is defined as Equ.(B.])

$$
I(i, j)=\frac{N_{o n}(m, n, l)}{N_{o f f}(m, n, l)}
$$

, where $N_{o n}(m, n, l)$ and $N_{o f f}(m, n, l)$ represent the events number at the local sidereal time (LST) the cell in $(\theta, \phi)$ space $(n, l)$ of the on- and off-source respectively. Since the relative intensities in the belt of the same zenith are the same, based on the principle of least squares, the $\chi^{2}$ equation can be constructed as Equ.(B.2)

$$
\chi^{2}(m, n, l)=\left(\frac{N_{o b s}(m, n, l)}{I(i, j)}-\frac{\sum_{l^{\prime} \neq l} \frac{N_{o b s}\left(m, n, l^{\prime}\right)}{I\left(i^{\prime}, j^{\prime}\right)}}{\sum_{l^{\prime} \neq l} 1}\right)^{2} \times\left(\frac{N_{o b s}(m, n, l)}{I^{2}(i, j)}+\frac{\sum_{l^{\prime} \neq l} \frac{N_{o b s}\left(m, n, l^{\prime}\right)}{I\left(i^{\prime}, j^{\prime}\right)}}{\left(\sum_{l^{\prime} \neq l} 1\right)^{2}}\right)^{-1}
$$

where $N_{o b s}(m, n, l)$ and $N_{o b s}\left(m, n, l^{\prime}\right)$ represents the observed events number in the on- and offsource region. From all the direction and at all the time, the total $\chi^{2}$ can be written as $\chi^{2}=$ $\sum_{(m, n, l)} \chi^{2}(m, n, l)$, which are solved by iteration. Then the distribution of relative intensities with declination and right ascension can be derived, as shown in the top of Fig.[.

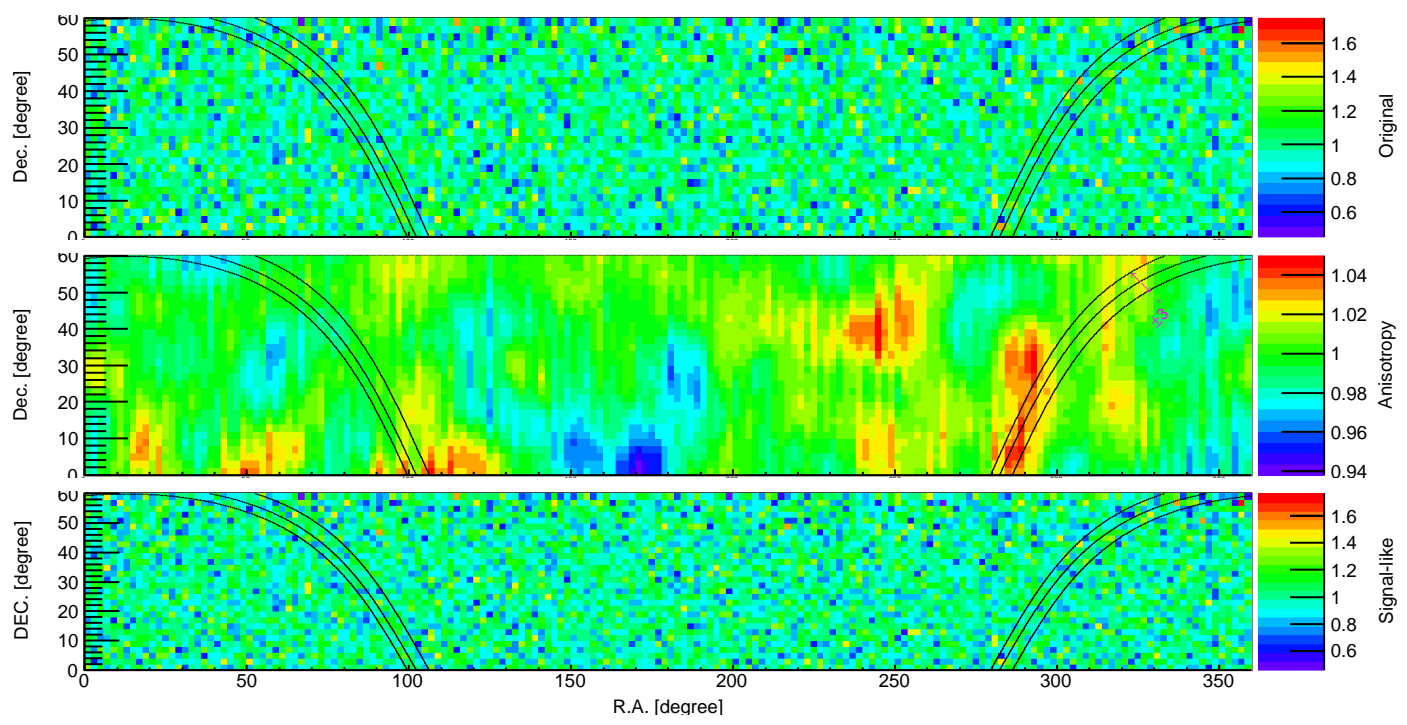

Figure 2: Relative intensity.

The galactic plane can be considered as an extended source. The excess event number and error of the excess can be derived by Equ.(B.3) and Equ.(B..4). Therefore, the significance of each 
cell $(\mathrm{i}, \mathrm{j})$ can be calculated by Equ.(3.5]).

$$
\begin{gathered}
N_{s}(i, j)=[I(i, j)-1] N(i, j) / I(i, j) \\
\Delta N_{s}(i, j)=\Delta I(i, j) N(i, j) / I(i, j) \\
S(i, j)=\frac{N_{s}(i, j)}{\Delta N_{s}(i, j)}
\end{gathered}
$$

\subsection{Large scale anisotropy}

In order to observe the $\gamma$-ray from the galactic plane, the large-scale anisotropy of CRs need to be deducted. As shown in Fig. . the solid line represents the location of the galactic plane. From the solid line region in Fig.】, it can be seen that though galactic plane is an extended region, it is small compare with the scale of CRs anisotropy. During the deduction, the signal region is excluded, the anisotropy background in a specific declination is estimated by averaging its surrounding $\pm 5^{\circ}$ declinations. Then the large-scale CRs anisotropy which is shown in the middle of Fig. subtracted, and the bottom of Fig.】 shows the relative intensity after the anisotropy deduction. Actually, it can be seen that the influence of the large-scael CRs anisotropy on the relative intensities of the $\gamma$-like events is quite small.

\section{Results and Summary}
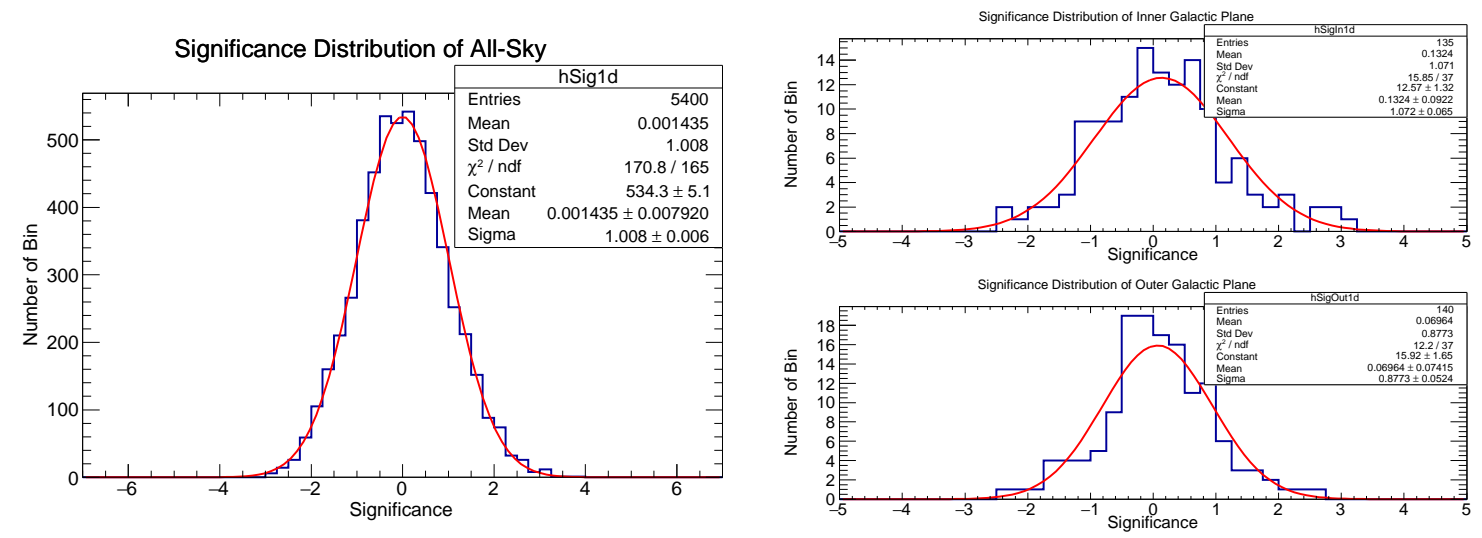

Figure 3: The left panel shows the significance level distribution of the all-sky, the right presents these from the inner and outer galactic plane.

Fig.B exhibits the significance level distributions, which are consistent with the Gaussian function. It indicates that no significant excess has been detected in this area by YBJ-HA. With the same analysis procedure, the data samples with sumpd range from 50 to 100 , whose primary energy is

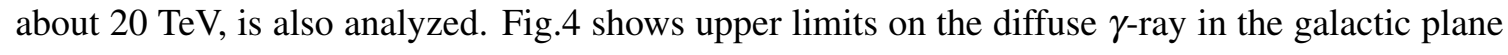
following the statistical method given by Helene[[18]. To reduce some systematic errors, upper limit on the flux ratio of $\gamma$-rays to CRs is also presented in Fig.[5. 

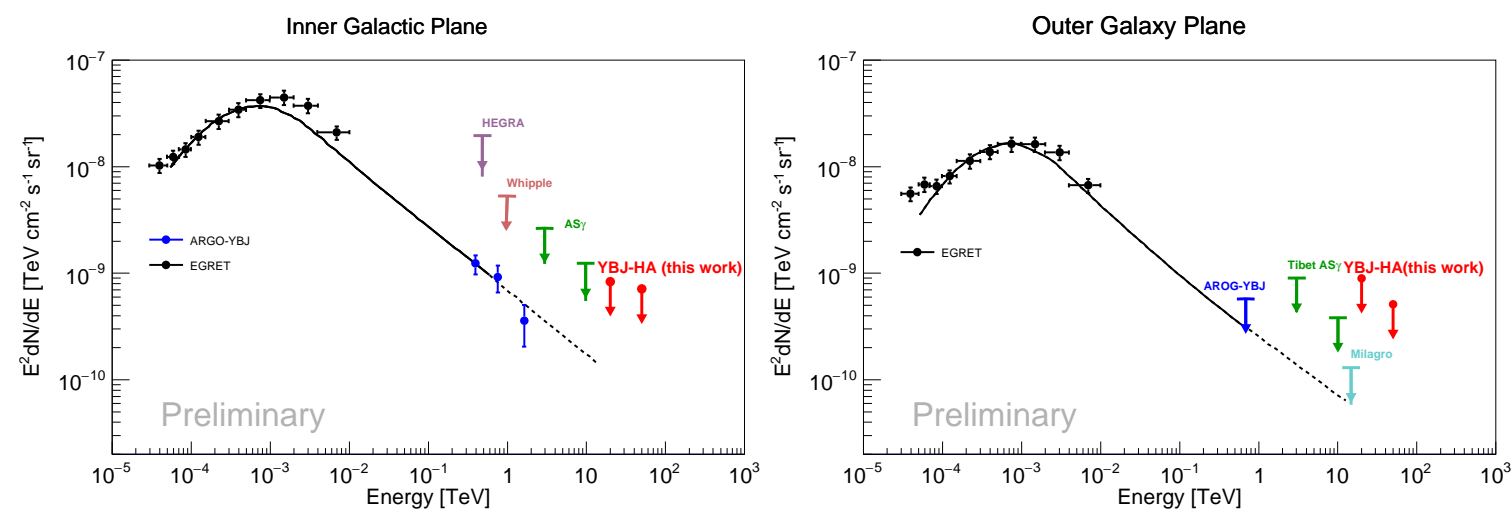

Figure 4: 90\% CL upper limits on the flux of the inner and outer galactic plane. Results from other experiments come from Ref.[प]]

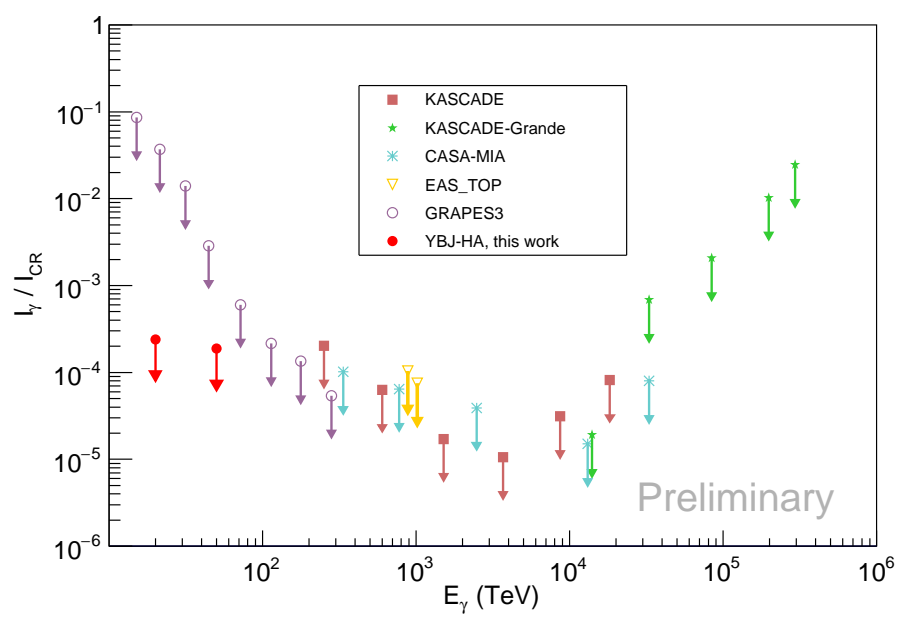

Figure 5: $90 \%$ CL upper limits on the ratio of $\gamma$-rays to CRs. Results from other experiments come from Ref.[ए]]

The preliminary analysis presented here shows that no significant excess on the observation of several dozens of $\mathrm{TeV} \gamma$-ray in the galactic plane observed by YBJ-HA in the first 160 days. We set 90\% CL upper limits on this region. As a pilot experiment of LHAASO experiment, the scale of the YBJ-HA is not large enough to capture gamma-ray emissions in such a short time duration. Even so YBJ-HA has been continuing collecting data, in addition, we foresee that the LHAASO will have a brighter further in the observation of $\gamma$-rays.

\section{Acknowledgements}

This work is supported by the Ministry of Science and Technology of China, National Key R\&D Program of China (2018YFA0404202) and Natural Sciences Foundation of China (11635011). 


\section{References}

[1] W. L. Kraushaar, G. W. Clark, G. P. Garmire, et al. High-Energy Cosmic Gamma-Ray Observations from the OSO-3 Satellite. ApJ, 177:341, November 1972.

[2] C. E. Fichtel, R. C. Hartman, D. A. Kniffen, et al. High-energy gamma-ray results from the second small astronomy satellite. ApJ, 198:163-182, May 1975.

[3] S. D. Hunter, D. L. Bertsch, J. R. Catelli, et al. EGRET Observations of the Diffuse Gamma-Ray Emission from the Galactic Plane. ApJ, 481:205-240, May 1997.

[4] M. Ackermann, M. Ajello, W. B. Atwood, et al. Fermi-LAT Observations of the Diffuse $\gamma$-Ray Emission: Implications for Cosmic Rays and the Interstellar Medium. ApJ, 750:3, May 2012.

[5] M. Ajello, A. Albert, W. B. Atwood, et al. Fermi-LAT Observations of High-Energy Gamma-Ray Emission toward the Galactic Center. ApJ, 819:44, March 2016.

[6] S. LeBohec, I. H. Bond, S. M. Bradbury, et al. Gamma-Ray Observations of the Galactic Plane at Energies E> 500 GEV. ApJ, 539:209-215, August 2000.

[7] F. A. Aharonian, A. G. Akhperjanian, J. A. Barrio, et al. A search for gamma-ray emission from the Galactic plane in the longitude range between $37^{\circ}$ and $43^{\circ}$. A\&A, 375:1008-1017, September 2001.

[8] A. A. Abdo, B. Allen, D. Berley, et al. Discovery of TeV Gamma-Ray Emission from the Cygnus Region of the Galaxy. ApJ, 658:L33-L36, March 2007.

[9] A. Abramowski, F. Aharonian, F. Ait Benkhali, et al. Diffuse Galactic gamma-ray emission with H.E.S.S. Phys. Rev. D, 90(12):122007, December 2014.

[10] B. Bartoli, P. Bernardini, X. J. Bi, et al. Study of the Diffuse Gamma-Ray Emission from the Galactic Plane with ARGO-YBJ. ApJ, 806:20, June 2015.

[11] F. Aharonian, A. G. Akhperjanian, A. R. Bazer-Bachi, et al. Discovery of very-high-energy $\gamma$-rays from the Galactic Centre ridge. Nature, 439:695-698, February 2006.

[12] HESS Collaboration, A. Abramowski, F. Aharonian, et al. Acceleration of petaelectronvolt protons in the Galactic Centre. Nature, 531:476-479, March 2016.

[13] Hershal Pandya and Zachary Griffith. Search for diffuse gamma-ray emission from the Galactic plane with IceCube. PoS, ICRC2017:705, 2018.

[14] Zhen Wang, Yiqing Guo, Hui Cai, et al. Performance of a scintillation detector array operated with lhaaso-km2a electronics. Experimental Astronomy, 45(3):363-377, Aug 2018.

[15] T. K. Gaisser, T. Stanev, and S. Tilav. Cosmic ray energy spectrum from measurements of air showers. Frontiers of Physics, 8(6):748-758, Dec 2013.

[16] J?rg R. H?randel. Models of the knee in the energy spectrum of cosmic rays. Astroparticle Physics, 21(3):241 - 265, 2004.

[17] M Amenomori, S Ayabe, D Chen, et al. A northern sky survey for steady tera-electron volt gamma-ray point sources using the tibet air shower array. The Astrophysical Journal, 633(2):1005-1012, 2005.

[18] O. Helene. Determination of the upper limit of a peak area. Nuclear Instruments and Methods in Physics Research Section A: Accelerators, Spectrometers, Detectors and Associated Equipment, 300(1):132 - 136, 1991.

[19] W. D. Apel, J. C. Arteaga-Velázquez, K. Bekk, et al. KASCADE-Grande Limits on the Isotropic Diffuse Gamma-Ray Flux between $100 \mathrm{TeV}$ and $1 \mathrm{EeV}$. ApJ, 848:1, October 2017. 